\title{
The level of IL-6 was associated with sleep disturbances in patients with major depressive disorder
}

This article was published in the following Dove Press journal:

Neuropsychiatric Disease and Treatment

\author{
Min Wang ${ }^{1,2}$ \\ Jinxue Wei ${ }^{1,2}$ \\ Xiao Yang ${ }^{1,2}$ \\ Peiyan $\mathrm{Ni}^{1,2}$ \\ Yingcheng Wang ${ }^{1,2}$ \\ Liansheng Zhao ${ }^{1,2}$ \\ Wei Deng ${ }^{1,2}$ \\ Wanjun Guo ${ }^{1,2}$ \\ Qiang Wang ${ }^{1,2}$ \\ Tao $\mathrm{Li}^{1,2}$ \\ Xiaohong $\mathrm{Ma}^{\mathrm{l}, 2}$ \\ 'Mental Health Center and Psychiatric \\ Laboratory, West China Hospital of \\ Sichuan University, Chengdu, People's \\ Republic of China; ${ }^{2}$ Huaxi Brain Research \\ Center, West China Hospital of Sichuan \\ University, Chengdu, People's Republic of \\ China
}

Correspondence: Xiaohong Ma Psychiatric Laboratory of West China Hospital, Sichuan University, I Keyuan 4th road, Chengdu, Sichuan 61004I, China

Email maxiaohong@scu.edu.cn
Purpose: Major depression disorder (MDD) was associated with inflammatory processes, but association results of inflammatory syndrome and MDD were inconsistent. To provide more evidence, we measured the plasma levels of IL-1 $\beta$, IL- 6 , interferon (INT)- $\alpha 2$, INT- $\gamma$, and tumor necrosis factor (TNF)- $\alpha$ in patients having MDD and explored correlations between the five proinflammatory cytokines and specific depressive symptoms.

Patients and methods: Plasma concentrations of IL- $1 \beta$, IL- 6 , INT- $\alpha 2$, INT- $\gamma$, and TNF- $\alpha$ were measured using ELISA for 44 MDD patients and 54 healthy controls. Patients with MDD were assessed on the 17-item Hamilton Depression Rating Scale (HAMD-17), and a total score and five syndrome scores were acquired.

Results: IL-6 levels in depressed patients were significantly elevated than in healthy controls, but no significant differences were observed in the levels of INF- $\alpha 2$, INF- $\gamma$, IL- $1 \beta$, or TNF- $\alpha$. In addition, correlation analysis revealed that sleep disturbances positively correlated with IL-6. Although there was no significant difference between the two groups in the levels of INF- $\alpha 2$, a significant positive correlation between IFN- $\alpha 2$ and retardation was observed.

Conclusion: Elevated IL-6 levels were observed in MDD patients and IL-6 may correlate with sleep disturbances.

Keywords: major depression disorder, inflammation, cytokine, interleukin-6, sleep disturbances

\section{Introduction}

Major depression disorder (MDD) is an enervating mental disorder that features depressed mood, slow thinking, and decreased volitional activity and is accompanied by sleep disorders, decreased appetite, and manifestations of autonomic nervous dysfunction. ${ }^{1}$ MDD has an overall impact on global disease and is predicted to become the leading burden of disease by the year $2020 .^{2}$ While effective treatments based on various neurotransmitter systems are available, approximately one-third of patients suffering from depression encompass a poor response to conventional antidepressant therapies. ${ }^{3}$ Consequently, it is urgent to develop new theoretical frameworks for understanding the development and progression of depression in order to formulate targeted and curative treatments. Accumulating evidence implicates that activation of the immune-inflammatory pathways is an important contributor to the development of MDD and shows the immune system as a feasible therapeutic target that may be more relevant to the pathogenesis of mood disorders in specific subpopulations. ${ }^{4-7}$ Inflammatory biomarkers have been demonstrated to cross the 
blood-brain barrier and thus interfere with a wide range of aspects of the pathophysiology of MDD, such as neuroplasticity, neurotransmitter metabolism, neuroendocrine function, and apoptosis of neurocyte. ${ }^{5,7,8}$ Nevertheless, numerous literature have shown that changes in the levels of inflammatory cytokine do not always present positive results in the development and treatment of depression. ${ }^{9,10}$ Given this, there is a hypothesis that inflammation is not prevalently present in, but only limited to, specific subgroups of individuals with MDD. ${ }^{11}$

Inflammation in depressed patients is a special pathological type since its inflammatory markers are not as elevated as those of bacterial infections. Some researchers hold that MDD is mainly induced by psychosocial stress in the subgroup undergoing chronic low-grade inflammation. Stressinduced neuroendocrine mechanisms, including glucocorticoid resistance (GR), ${ }^{4}$ the hypothalamic-pituitary-adrenal (HPA) axis hyperactivity, ${ }^{12}$ and the sympathetic nervous system (SNS)-mediated dysfunction, ${ }^{13}$ may function as pivotal immunological interfaces between stress and inflammation. In a stressful environment, central nervous fibers release the neurotransmitters into lymphoid organs and peripheral tissues in which inflammatory reactions take place, ${ }^{12}$ and hypothalamus cytokine receptors trigger the production of glucocorticoids by activating the HPA axis. ${ }^{13}$ A research showed that psychological changes associated with acclimatization to stress dynamics are persistent and cumulative changes occur in the structure of the CNS. ${ }^{14}$ Stress-induced GR is a typical biological abnormality and has been correlated with increased inflammation in depressed patients. ${ }^{15}$ In addition, there are a good deal of studies that reported increased levels of proinflammatory cytokines, including IL-6, IL-1 $\beta$, interferon (INT)- $\alpha$, and tumor necrosis factor (TNF)- $\alpha$ in depressed patients. ${ }^{16-19}$ Noteworthily, researches have shown that IL-6 is involved in a variety of physiological systems, including the activation of HPA axis, corticotrophin-releasing hormone activity, apoptotic pathways and the induction of oxidative stress, ${ }^{4-7,12,13}$ all of which are relevant to the pathophysiology of MDD. In general, these data support the assumption that the inflammatory cytokines may be critical immunological points for the connection of stress-induced risk signals that ultimately drive inflammatory responses related to depression.

In addition, an association has been observed between chronic low-grade inflammation and the severity of depression symptom and even particular depressive psychopathology. $^{20-23}$ For instance, compelling evidence has demonstrated that lower sleep efficiency is associated with elevated inflammatory biomarkers. ${ }^{23}$ A study showed that peripheral inflammatory markers, including elevated IL6 and monocyte chemotactic protein, were consistently associated with psychomotor speed in MDD. ${ }^{16}$ Another study showed that increased appetite was related to C-reactive protein (CRP) and TNF- $\alpha$ in the context of a depressive episode. $^{24}$ In a prospective study involving 190 patients who had contracted hepatitis $\mathrm{C}$ virus and initiation of standard treatment with IFN- $\alpha$, the authors found that during receiving chronic IFN- $\alpha$ treatment, the rate of meeting diagnostic criteria for MDD was up to $50 \%$, and psychomotor retardation and somatic symptoms may reflect a greater inflammatory state before treatment. ${ }^{20}$ Nevertheless, some symptoms may be more strongly correlated to inflammation than others, some may be the result of inflammation, and others may indicate increases in inflammation. ${ }^{25}$ Despite the large bulk of literature linking depression with inflammation, the associations of inflammation with specific depression symptoms remain largely unknown. In addition, only few clinical research involving patients with mood disorders have examined whether proinflammatory cytokines were differently related to specific depression symptoms and the results were inconsistent.

Therefore, to investigate cytokines in patients with MDD, we examined the concentrations of the proinflammatory cytokines IL- $1 \beta$, IL- 6 , TNF- $\alpha$, INF- $\alpha 2$, and INF- $\gamma$ in patients with moderate-to-major depression. What is more, we aimed to investigate the relationships between specific depression symptoms and proinflammatory cytokines.

\section{Methods}

\section{Subjects}

The study involved 44 patients with depression and 54 sexand age-matched healthy controls who were recruited from the Mental Health Center of West China Hospital of Sichuan University. Participants were Han Chinese aged between 18 and 60 years who were physically healthy with Wechsler's intelligence test scores being $>90$. The diagnosis was consistent with the criteria for MDD according to the Diagnostic and Statistical Manual of Mental Disorders, Fourth Edition (DSM-IV). The severity of depression was assessed using the 17-item Hamilton Depression Rating Scale (HAMD-17), ${ }^{26}$ which evaluated symptoms in five dimensions, namely anxiety, weight, cognition, slowness, and sleep disorder. The total scores of HAMD-17>17 were obtained. The 14-item Hamilton anxiety rating scale (HAMA-14) was also used for evaluation. 
Participants were excluded if they: 1) had neurogenic diseases or a history of endocrine, autoimmune, metabolic disorders; 2) had other DSM-IV axis I and II diseases, such as bipolar disorder, schizophrenia, substance abuse, or a family history of mental illness; 3 ) were taking hormone, antibiotics, or anti-inflammatory medications; or 4) were pregnant or lactating.

All participants provided informed written consent. The study was approved by the Institutional Ethics Committee of Sichuan University. All study procedures were in accordance with the Declaration of Helsinki.

\section{Cytokine detections}

Peripheral blood of all participants was collected by venipuncture. The samples were placed in EDTA anticoagulant tubes for laboratory tests. Centrifugation was performed at $2,000 \times \mathrm{g}$ for 5 mins. After that, the upper plasma was moved to a fresh tube and then stored at $-80^{\circ} \mathrm{C}$. A multiplexed flow cytometric assay using a Milliplex kit (HCYTOMAG-60K; Millipore, Billerica, MA, USA) was applied to measure the concentrations of proinflammatory cytokines including IL- $1 \beta$, IL- 6 , TNF- $\alpha$, INF- $\alpha$, and INF- $\gamma$. Their levels were expressed in $\mathrm{pg} / \mathrm{mL}$. The assays were conducted by Bio-atom Biotechnology (Chengdu, China) following the instructions provided by the manufacturer.

\section{Statistical analysis}

Statistical analysis was performed by means of the SPSS 25.0 software package. All data are presented as mean $\pm \mathrm{SE}$ unless otherwise stated. $\chi^{2}$ test was used to compare categorical variables for demographic and clinical characteristics. Student's $t$-test was applied for continuous variables. The Mann-Whitney U test was applied to compare the concentrations since the concentrations of cytokine in plasma did not follow a Gaussian distribution. The Spearman correlation test was used to ascertain the associations between the cytokines' concentrations and the five syndrome scores of the HAMD-17. All P-values $<0.05$ were considered statistically significant. We did not correct for multiple testing.

\section{Results}

\section{Demographic and clinical characteristics of the participants}

The study included 44 subjects with depression (20 men and 24 women) and 54 healthy individuals (19 men and 35 women). The average age of the participants having MDD and healthy controls was $29.27 \pm 10.83$ and $29.67 \pm 10.29$ years, respectively. Age $(P=0.86)$, gender $(P=0.41)$, and body mass index (BMI) $(P=0.158)$ between the two groups were not different between depressed patients and their controls. The total HAMD and HAMA scores of all the participants having MDD were $22.60 \pm 4.93$ and $14.44 \pm 6.88$, respectively (Table 1).

\section{Plasma levels of IL-I $\beta$, IL-6, TNF- $\alpha$, INF- $\alpha$, and INF- $\gamma$}

The plasma concentrations of IL-1 $\beta$, IL-6, TNF- $\alpha$, INF- $\alpha 2$, and INF- $\gamma$ in all participants are presented (Table 2). The levels of IL-6 were significantly higher in MDD patients than in controls $(P=0.031)$, while the differences for IL-1 $\beta$, TNF- $\alpha$, INF- $\alpha 2$, and INF- $\gamma$ were not significant $(P=0.627$, $P=0.178, P=0.347$, and $P=0.169$, respectively).

\section{Associations between pro-inflammatory cytokines and specific depression symptoms in MDD patients}

A significantly positive association was observed between IL-6 and sleep disturbance among MDD patients $(r=0.329$,

Table I Demographic and clinical characteristics of 98 participants

\begin{tabular}{|l|l|l|l|}
\hline \multirow{2}{*}{ Characteristics } & $\begin{array}{l}\text { MDD } \\
\text { patients }\end{array}$ & HC & P \\
\cline { 2 - 3 } & $\mathbf{( n = 4 4 )}$ & $\mathbf{( n = 5 4 )}$ & \\
\hline Males (n) & 20 & 19 & 0.407 \\
Age (yesrs) & $29.27 \pm 10.83$ & $29.67 \pm 10.29$ & 0.855 \\
Education year & $13.11 \pm 3.05$ & $14.80 \pm 3.84$ & 0.02 \\
BMI & $20.73 \pm 3.06$ & $21.63 \pm 3.18$ & 0.158 \\
Age of onset (years) & $27.35 \pm 10.67$ & - & - \\
Number of episode & $1.79 \pm 2.21$ & - & - \\
Disease duration (months) & $50.7 \pm 64.80$ & - & - \\
Duration the current epi- & $4.88 \pm 5.01$ & - & - \\
sode (months) & & & \\
HAMA total scores & $14.44 \pm 6.88$ & - & - \\
HAMDI7 total scores & $22.60 \pm 4.93$ & - & - \\
Anxiety/somatization & $4.65 \pm 1.63$ & - & - \\
(scores) & & & - \\
Weight (scores) & $1.16 \pm 0.90$ & - & - \\
Cognitive disturbance & $2.44 \pm 1.61$ & - & - \\
(scores) & & & - \\
Retardation (scores) & $3.58 \pm 1.50$ & - & - \\
Sleep disturbance (scores) & $6.70 \pm 2.64$ & - & - \\
\hline Notes: Dan preseredas & & - \\
\hline
\end{tabular}

Notes: Data presented as mean \pm standard deviation unless otherwise stated. Anxietyl somatization, HAMD 10, 11, 12, 15, 17; Weight, HAMD 16; Cognitive disturbance, HAMD 2, 3, 9; Retardation, HAMD I, 7, 8, I4; Sleep disturbance, HAMD 4, 5, 6.

Abbreviations: MDD, major depressive disorder; $\mathrm{HC}$, healthy control; BMI, body mass index; HAMA, Hamilton Anxiety Rating Scale; HAMD, Hamilton Depression Rating Scale. 
Table 2 The median of five cytokines concentration in patients with MDD and healthy controls

\begin{tabular}{|l|l|l|l|l|}
\hline $\begin{array}{l}\text { Cytokine } \\
\text { (pg/mI) }\end{array}$ & $\begin{array}{l}\text { MDD patients } \\
(\mathbf{n = 4 4 )}\end{array}$ & $\begin{array}{l}\text { HC } \\
(\mathbf{n}=\mathbf{5 4})\end{array}$ & $\mathbf{Z}$ & $\boldsymbol{P}$ \\
\hline IL-I $\beta$ & 1.73 & 1.59 & -0.87 & 0.627 \\
IL-6 & 1.09 & 0.82 & -2.113 & $0.03 \mathrm{I}^{\mathrm{a}}$ \\
TNF- $\alpha$ & 7.05 & 6.21 & -0.802 & 0.178 \\
IFN- $\alpha 2$ & 5.2 & 3.62 & -0.859 & 0.347 \\
IFN- $\gamma$ & 12.94 & 9.87 & -0.866 & 0.169 \\
\hline
\end{tabular}

Notes: ${ }^{a} P<0.05$.

Abbreviations: MDD: Major Depressive Disorder; HC: Healthy control; IL, interleukin; TNF, tumor necrosis factor; IFN, interferon.

$P=0.031$ ) (Table 3). Nevertheless, no significant difference in IFN- $\alpha 2$ levels was observed between patients with MDD and healthy controls. However, a significant correlation between IFN- $\alpha 2$ and retardation was found.

\section{Discussion}

Our results documented that the level of IL-6 was increased in patients with MDD when compared with healthy controls. However, the levels of INF- $\alpha 2$, INF- $\gamma$, IL-1 $\beta$, and TNF- $\alpha$ were not significantly different between the two groups. The association between higher IL-6 and sleep disturbance was significant among MDD patients. A significant difference in IFN- $\alpha 2$ levels was observed between patients with MDD and healthy controls. Interestingly, we also found a significant correlation between IFN- $\alpha 2$ and retardation.

Our results regarding IL-6 were consistent with a number of prior reports by other researchers. ${ }^{16-19}$ A previous study showed that the concentrations of the serum IL-6 in depressed patients were significantly higher than that in the controls. ${ }^{16}$ A recent meta-analysis involving 42 studies supported this finding, with significantly elevated IL-6 levels in participants with MDD compared with healthy controls. ${ }^{9}$ A more recent meta-analysis also suggested that the concentrations of IL- 6 in cerebrospinal fluid in individuals with MDD were higher than those in controls. $^{27}$ An increase of IL-6 in peripheral blood may reflect a relative sensitized inflammatory response, which may suggest a state of mild inflammation at the brain levels. ${ }^{7,8}$ Another longitudinal study manifested that elevated IL-6 increases the risk for depressive symptoms over time. ${ }^{28}$ Inflammation may also give rise to treatment nonresponse by affecting the neurotransmitter systems associated with antidepressant efficacy, and higher IL-6 levels were proved to be associated with a greater number of treatment failures. $^{29}$

However, the IL-6 levels changes were inconsistent in patients with MDD. Some studies found there was no difference in IL-6 levels between patients with MDD and their controls. ${ }^{19,20,30}$ One possible explanation for such inconsistency was that inflammatory marker levels in people with major depression may be higher than those with mild depression. ${ }^{31}$ This possibility helps explain why IL-6 levels were significantly higher in MDD patients than in controls in the present study: our sample consisted primarily of patients with moderate-to-severe depression.

Multiple lines of evidence have shown that inflammatory markers are associated with specific depressive symptoms. Our results confirm that lower sleep efficiency was associated with higher circulating levels of IL-6 in midlife women. ${ }^{23}$ Elevated inflammatory cytokines in patients can lead to various forms of sleep disturbance in other diseases, such as schizophrenia, ${ }^{32}$ sleep apnea-hypopnea syndrome (SAHS), ${ }^{33}$ type 2 diabetes, and cardiovascular events. ${ }^{34}$ Because inflammatory responses can be triggered by obesity, hypoxia, and hyperlipidemia, levels of proinflammatory factors may be elevated. One thorny question is whether inflammation causes sleep disorders or whether sleep disorders cause inflammation. Based on our study, we propose that for patients with elevated inflammatory

Table 3 Relationship between cytokine concentrations and the five syndrome scores

\begin{tabular}{|l|l|l|l|l|l|l|l|l|l|l|l|}
\hline \multirow{2}{*}{ Cytokine } & \multicolumn{2}{l|}{ Anxiety/somatization } & \multicolumn{2}{l|}{ Weight } & \multicolumn{2}{l|}{ Cognitive disturbance } & \multicolumn{2}{l|}{ Retardation } & \multicolumn{2}{l|}{ Sleep disturbance } \\
\cline { 2 - 11 } & $\mathbf{r}$ & $\boldsymbol{P}$ & $\mathbf{r}$ & $\boldsymbol{P}$ & $\mathbf{r}$ & $\boldsymbol{P}$ & $\mathbf{r}$ & $\boldsymbol{P}$ & $\mathbf{r}$ & $\boldsymbol{P}$ \\
\hline IL-I $\beta$ & $-0.1 \mathrm{II}$ & 0.477 & -0.108 & $0.49 \mathrm{I}$ & 0.034 & 0.828 & 0.186 & 0.233 & 0.069 & 0.662 \\
IL-6 & -0.15 & 0.337 & 0.024 & 0.877 & 0.095 & 0.543 & 0.038 & $0.81 \mathrm{I}$ & 0.329 & $0.03 \mathrm{I}^{\mathrm{a}}$ \\
TNF $\alpha$ & -0.164 & 0.293 & $0.07 \mathrm{I}$ & $0.65 \mathrm{I}$ & 0.115 & 0.464 & 0.108 & 0.492 & 0.184 & 0.237 \\
IFN- $\alpha 2$ & $-0.0 \mathrm{II}$ & 0.944 & -2.1 & 0.177 & -0.22 & 0.89 & 0.344 & $0.024^{\mathrm{a}}$ & 0.118 & 0.451 \\
IFN- $\gamma$ & -0.077 & 0.624 & $0.08 \mathrm{I}$ & 0.606 & 0.029 & 0.853 & -0.069 & 0.658 & 0.054 & 0.73 \\
\hline
\end{tabular}

Notes: ${ }^{a} P<0.05 ; r$, Spearman correlation coefficient.

Abbreviations: IL, interleukin; TNF, tumor necrosis factor; IFN, interferon. 
cytokines, medical staff should be aware of the occurrence of sleep disorders. Large prospective studies should be conducted to provide stronger evidence to support the inflammatory hypothesis of depression.

Our study also found a significant correlation between IFN- $\alpha 2$ and retardation, although no difference was observed in the level of INF- $\alpha 2$ between MDD patients and healthy controls. Our findings are consistent with the study in which no difference was found between MDD patients and healthy controls. ${ }^{35}$ INF- $\alpha 2$ and IFN- $\gamma$ are both proinflammatory factors in the INT family. In our study, no difference in the levels of INF- $\gamma$ was found between the two groups. A meta-analysis involving 17 studies indicated that the levels of IFN- $\gamma$ were reduced in patients with MDD compared to the healthy controls. ${ }^{9}$ We found a significantly positive correlation between IFN- $\alpha 2$ and retardation. Previous studies have shown that IFN- $\alpha$ injections may trigger depression in patients infected with hepatitis c virus, and psychomotor retardation, and somatic symptoms may play important roles. ${ }^{20}$

\section{Limitations}

However, this study has several weaknesses that warrant consideration. First, the study was not representative enough because it had a small sample size, and all patients exhibited a similar severity of moderate-to-severe depression. These two factors decreased the study's statistical power, and we did not correct for multiple testing. Therefore, it is better for future studies to involve a larger sample size given the heterogeneity of clinical manifestations of depression. ${ }^{11,36}$ Second, the crosssectional study did not allow us to draw any conclusions regarding cause-to-effect relationships. Longitudinal studies should be performed to observe the causal relationships between inflammation and specific depressive symptoms. Finally, the scale was simplified, and the description of symptoms was not comprehensive and accurate enough. Therefore, future studies can evaluate depressive symptoms using multiple scales simultaneously, such as the Montgomery depression scale and the selfrating depression scale.

\section{Conclusion}

In summary, elevated IL-6 level is observed in MDD patients. Besides, IL-6 may correlate with sleep disturbance. Further investigation may expand the sample size and increase scale category to confirm the results.

\section{Acknowledgments}

This research was supported by the National Natural Science Foundation of China (Grant No.: 81671344), and the 1.3.5 Project for Disciplines of Excellence, West China Hospital of Sichuan University (Grant No.: ZY2016103 and ZY2016203).

\section{Author contributions}

MW, XM, JW, and XY designed the study. MW performed the statistical analyses and wrote the initial draft of the manuscript. All of the authors contributed to data collection and analysis, critically revised the article and gave final approval of the version to be published, and agreed to be accountable for all aspects of the work in ensuring that questions related to the accuracy or integrity of any part of the work are appropriately investigated and resolved.

\section{Disclosure}

The authors report no conflicts of interest in this work.

\section{References}

1. American Psychiatric Association (APA). [DSM-5. Diagnostic and Statistical Manual of Mental Disorders] Manual Diagnóstico $Y$ Estadístico De Los Trastornos Mentales. DSM-5® EAN: 9788498358100. 5a. Washington, DC: American Psychiatric Association; 2014

2. Lasalvia A, Zoppei S, Van Bortel T, et al. Global pattern of experienced and anticipated discrimination reported by people with major depressive disorder: a cross-sectional survey. Lancet. 2013;381 (9860):55-62. doi:10.1016/S0140-6736(12)61379-8

3. Rush AJ, Trivedi MH, Wisniewski SR, et al. Acute and longer-term outcomes in depressed outpatients requiring one or several treatment steps: a STAR*D report. Am J Psychiatry. 2006;163(11):1905-1917. doi:10.1176/ajp.2006.163.11.1905

4. Pace TWW, Fang H, Miller AH. Cytokine-effects on glucocorticoid receptor function: relevance to glucocorticoid resistance and the pathophysiology and treatment of major depression. Brain Behav Immun. 2007;21(1):9-19. doi:10.1016/j.bbi.2006.08.009

5. Dantzer R, O'Connor JC, Freund GG, Johnson RW, Kelley KW. From inflammation to sickness and depression: when the immune system subjugates the brain. Nat Rev Neurosci. 2008;9(1):46-56. doi:10.1038/nrn2297

6. Iwata M, Ota KT, Duman RS. The inflammasome: pathways linking psychological stress, depression, and systemic illnesses. Brain Behav Immun. 2013;31:105-114. doi:10.1016/j.bbi.2012.12.008

7. Miller AH, Raison CL. The role of inflammation in depression: from evolutionary imperative to modern treatment target. Nat Rev Immunol. 2016;16(1):22-34. doi:10.1038/nri.2015.5

8. Yarlagadda A, Alfson E, Clayton AH. The blood brain barrier and the role of cytokines in neuropsychiatry. Psychiatry. 2009;6(11):18-22.

9. Hiles SA, Baker AL, de Malmanche T, Attia J. Interleukin-6, C-reactive protein and interleukin-10 after antidepressant treatment in people with depression: a meta-analysis. Psychol Med. 2012;42 (10):2015-2026. doi:10.1017/S0033291712000128

10. Kohler CA, Freitas TH, Maes M, et al. Peripheral cytokine and chemokine alterations in depression: a meta-analysis of 82 studies. Acta Psychiatr Scand. 2017;135(5):373-387. doi:10.1111/acps.12698

11. Rantala MJ, Luoto S, Krams I, Karlsson H. Depression subtyping based on evolutionary psychiatry: proximate mechanisms and ultimate functions. Brain Behav Immun. 2018;69:603-617. doi:10.1016/ j.bbi.2017.10.012 
12. Lamers F, Vogelzangs N, Merikangas KR, de Jonge P, Beekman AT, Penninx BW. Evidence for a differential role of HPA-axis function, inflammation and metabolic syndrome in melancholic versus atypical depression. Mol Psychiatry. 2013;18(6):692-699. doi:10.1038/ mp.2012.144

13. Irwin MR, Cole SW. Reciprocal regulation of the neural and innate immune systems. Nat Rev Immunol. 2011;11(9):625-632. doi:10.1038/nri3042

14. Post RM, Fleming J, Kapczinski F. Neurobiological correlates of illness progression in the recurrent affective disorders. J Psychiatr Res. 2012;46(5):561-573. doi:10.1016/j.jpsychires.2012.02.004

15. Maslanik T, Tannura K, Mahaffey L, et al. Commensal bacteria and MAMPs are necessary for stress-induced increases in IL-1beta and IL-18 but not IL-6, IL-10 or MCP-1. PLoS One. 2012;7(12):e50636. doi:10.1371/journal.pone.0050636

16. Yang K, Xie G, Zhang Z, et al. Levels of serum interleukin (IL)-6, IL-1beta, tumour necrosis factor-alpha and leptin and their correlation in depression. Aust $N Z Z J$ Psychiatry. 2007;41(3):266-273. doi:10.1080/00048670601057759

17. Al-Hakeim HK, Al-Rammahi DA, Al-Dujaili AH. IL-6, IL-18, sIL-2R, and TNFalpha proinflammatory markers in depression and schizophrenia patients who are free of overt inflammation. $J$ Affect Disord. 2015;182:106-114. doi:10.1016/j.jad.2015.04.044

18. Bahrini L, Ouanes S, Ghachem R. Inflammatory profile in depression and associated clinical and sociodemographic features in a Middle-Eastern North-African population. $J$ Affect Disord. 2016;198:122-126. doi:10.1016/j.jad.2016.03.036

19. Zou W, Feng R, Yang Y. Changes in the serum levels of inflammatory cytokines in antidepressant drug-naive patients with major depression. PLoS One. 2018;13(6):e0197267. doi:10.1371/journal.pone.0197267

20. Whale R, Fialho R, Rolt M, et al. Psychomotor retardation and vulnerability to interferon alpha induced major depressive disorder: prospective study of a chronic hepatitis C cohort. J Psychosom Res. 2015;79(6):640-645. doi:10.1016/j.jpsychores.2015.06.003

21. Goldsmith DR, Haroon E, Woolwine BJ, et al. Inflammatory markers are associated with decreased psychomotor speed in patients with major depressive disorder. Brain Behav Immun. 2016;56:281-288. doi:10.1016/j.bbi.2016.03.025

22. Chu AL, Stochl J, Lewis G, Zammit S, Jones PB, Khandaker GM. Longitudinal association between inflammatory markers and specific symptoms of depression in a prospective birth cohort. Brain Behav Immun. 2019;76:74-81. doi:10.1016/j.bbi.2018.11.007

23. Nowakowski S, Matthews KA, von Kanel R, Hall MH, Thurston RC. Sleep characteristics and inflammatory biomarkers among midlife women. Sleep. 2018;41(5). doi:10.1093/sleep/zsy024

24. Lamers F, Milaneschi Y, de Jonge P, Giltay EJ, Penninx B. Metabolic and inflammatory markers: associations with individual depressive symptoms. Psychol Med. 2018;48(7):1102-1110. doi:10.1017/ S0033291717002483
25. Khandaker GM, Stochl J, Zammit S, Goodyer I, Lewis G, Jones PB. Childhood inflammatory markers and intelligence as predictors of subsequent persistent depressive symptoms: a longitudinal cohort study. Psychol Med. 2018;48(9):1514-1522. doi:10.1017/ S0033291717003038

26. Hamilton M. A rating scale for depression. J Neurol Neurosurg Psychiatry. 1960;23(1):56-62. doi:10.1136/jnnp.23.1.56

27. Wang AK, Miller BJ. Meta-analysis of cerebrospinal fluid cytokine and tryptophan catabolite alterations in psychiatric patients: comparisons between schizophrenia, bipolar disorder, and depression. Schizophr Bull. 2017;44:1.

28. Valkanova V, Ebmeier KP, Allan CL. CRP, IL-6 and depression: a systematic review and meta-analysis of longitudinal studies. J Affect Disord. 2013;150(3):736-744. doi:10.1016/j.jad.2013.06.004

29. Haroon E, Daguanno AW, Woolwine BJ, et al. Antidepressant treatment resistance is associated with increased inflammatory markers in patients with major depressive disorder. Psychoneuroendocrinology. 2018;95:43-49. doi:10.1016/j.psyneuen.2018.05.026

30. Hocaoglu C, Kural B, Aliyazicioglu R, Deger O, Cengiz S. IL-1beta, IL-6, IL-8, IL-10, IFN-gamma, TNF-alpha and its relationship with lipid parameters in patients with major depression. Metab Brain Dis. 2012;27(4):425-430. doi:10.1007/s11011-012-9323-9

31. Kohler-Forsberg O, Buttenschon HN, Tansey KE, et al. Association between $\mathrm{C}$-reactive protein (CRP) with depression symptom severity and specific depressive symptoms in major depression. Brain Behav Immun. 2017;62:344-350. doi:10.1016/j.bbi.2017.02.020

32. Lee EE, Ancoli-Israel S, Eyler LT, et al. Sleep disturbances and inflammatory biomarkers in schizophrenia: focus on sex differences. Am J Geriatr Psychiatry. 2019;27(1):21-31. doi:10.1016/j. jagp.2018.09.017

33. Lopez-Pascual A, Lasa A, Portillo MP, et al. Low oxygen consumption is related to a hypomethylation and an increased secretion of IL-6 in obese subjects with sleep apnea-hypopnea syndrome. Ann Nutr Metab. 2017;71(1-2):16-25. doi:10.1159/000478276

34. Meng LL, Tang YZ, Ni CL, et al. Impact of inflammatory markers on the relationship between sleep quality and incident cardiovascular events in type 2 diabetes. J Diabetes Complications. 2015;29 (7):882-886. doi:10.1016/j.jdiacomp.2015.06.011

35. Chen Y, Ouyang J, Liu S, Zhang S, Chen P, Jiang T. The role of cytokines in the peripheral blood of major depressive patients. Clin Lab. 2017;63(7):1207-1212. doi:10.7754/Clin.Lab.2017.170117

36. Ruiz NAL, Del Angel DS, Olguin HJ, Silva ML. Neuroprogression: the hidden mechanism of depression. Neuropsychiatr Dis Treat. 2018;14:2837-2845. doi:10.2147/NDT.S177973
Neuropsychiatric Disease and Treatment

\section{Publish your work in this journal}

Neuropsychiatric Disease and Treatment is an international, peerreviewed journal of clinical therapeutics and pharmacology focusing on concise rapid reporting of clinical or pre-clinical studies on a range of neuropsychiatric and neurological disorders. This journal is indexed on PubMed Central, the 'PsycINFO' database and CAS, and is the official journal of The International Neuropsychiatric Association (INA). The manuscript management system is completely online and includes a very quick and fair peer-review system, which is all easy to use. Visit http://www.dovepress.com/testimonials.php to read real quotes from published authors. 